\title{
Minimal Adhesion Surface Area In Tangentially Loaded Digital Contacts
}

\author{
Alexander V. Terekhov, Vincent Hayward* \\ UPMC Univ Paris 06, UMR 7222, \\ Institut des Systèmes Intelligents et de Robotique, Paris, France
}

\begin{abstract}
The stick-to-slip transition of a fingertip in contact with a planar surface does not occur instantaneously. As the tangential load increases, portions of the skin adhere while others slip, giving rise to an evolution of the contact state, termed partial slip. We develop a quasi-static model that predicts that if the coefficient of kinetic friction is larger than the coefficient of static friction, then the stuck surface area diminishes as the tangential load increases until reaching a 'minimal adhesion surface area' where it vanishes abruptly. This phenomenon was observed in recently measured finger-slip image data (André et al., 2011) that were processed by an optic flow detection algorithm. We examined the results of ten trials. Four of them exhibited the minimal adhesion surface area phenomenon, four of them did not, and two were inconclusive.
\end{abstract}

Keywords: Fingertip contact mechanics, Fingertip slip dynamics, Fingers.

Word count: 1500 .

\section{Introduction}

Studies about manual prehension implicitly assume that fingertips either adhere during a secure grip or that they slip (Cole and Johansson, 1993; Witney et al., 2004; Aoki et al., 2006; Zatsiorsky and Latash, 2008). This sharp distinction is also often assumed in order to describe purposeful touch (Smith

\footnotetext{
*Corresponding author

Email address: vincent.hayward@isir.upmc.fr (Vincent Hayward)
} 
et al., 2002; Hayward, 2008). Imaging studies, however, showed that transitions from adhesion to slip are far from being instantaneous. They result from a process developing through time (Levesque and Hayward, 2003; Tada and Kanade, 2004; André et al., 2011).

On flat surfaces, the total contact area is divided into a shrinking, stuck region and a growing, slip region. Moreover, the stuck region remains connected and completely contained within the slip region (Fig. 1). The contact state between full adhesion and full slip is termed partial slip.

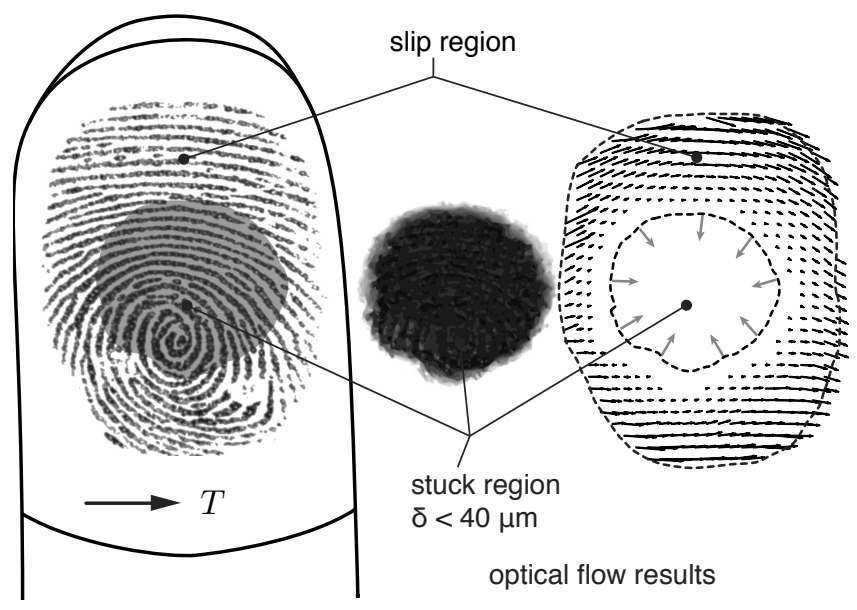

Figure 1: Fingertip contact imaging and optic flow processing (Levesque and Hayward, 2003; Bruhn et al., 2005). Arrows show displacements. The stuck region was where displacement was below $40 \mu \mathrm{m}$ (one pixel). Relative stuck surface area, $d^{2}$, was computed from the number of pixels in the stuck region and in the contact region after contour extraction (dashed lines).

While it is tempting to study the complete dynamics of partial slip, in the absence of pronounced stick-slip oscillations, it is sufficient to study the evolution of a quasi-static problem on the grounds that masses, accelerations, and velocities are sufficiently small for inertial and viscous forces to be neglected in the face of elastic forces. Time can then be eliminated, and the contact evolution studied as a function of the load supported by the contact. This view is adopted in classic theoretical studies in contact mechanics (for example, Johnson, 1955).

The dependency of the stuck surface area on the tangential load could be thought to have one of two forms, see Fig 2a. Either the stuck region 
diminishes continuously as the load increases, or it diminishes until reaching a certain critical size at which point it vanishes, since we exclude an instantaneous transition from full adhesion to slip.

Here, we examine the hypothesis that, under increasing load, not all stuck surfaces continuously diminish until reaching a zero area. Specifically, if the static coefficient of friction is greater than the kinetic coefficient of friction, then there exists a minimal stuck surface area. From considerations regarding the statics of distributed traction, we compared the predictions of a newly introduced model with experimental data acquired in a recent study where finger contacts were imaged while the load was measured during the course of finger slips on glass surfaces (André et al., 2011), and found evidence of the occurrence of this phenomenon.

\section{Methods}

\subsection{Model}

The model assumes that the pressure distribution, $p(x, y)$, inside the contact region can be represented by a quadratic function which, according to Pawluk and Howe (1999), well approximates experimental data. The pressure distribution takes the form,

$$
p(x, y)=\frac{2 N}{\pi a b}\left[1-\left(\frac{x^{2}}{a^{2}}+\frac{y^{2}}{b^{2}}\right)\right],
$$

in an elliptical contact region, with $p=0$ at its border. For any parameters, $a, b$, the integral over the contact is equal to the total normal load, $N$.

In the absence of pivoting, it is reasonable to assume that the traction, $q(x, y)$, is uniform in the stuck region, which is to assume that the tangential elasticity is uniformly distributed. This assumes that the support boundary condition (the bone and nail) is sufficiently far from the region of contact to have a uniform effect. Under this assumption, if $\mu_{k}$ is the coefficient of kinetic friction,

$$
q(x, y)= \begin{cases}q_{\mathrm{stick}}, & \text { in the stuck region, } \\ \mu_{k} p(x, y), & \text { in the slip region. }\end{cases}
$$

Note that if the presliding distance of the skin contact can be considered to be small in comparison to the net finger displacement at each point of the 
contact, ${ }^{1}$ then discontinuities in the traction are lawful.

The adhesion condition, $q_{\text {stick }} \leq \mu_{s} p(x, y)$, where $\mu_{s}$ is the coefficient of static friction, must be satisfied inside the stuck region. On the boundary, which is approximated by an ellipse, $q_{\text {stick }}=\mu_{s} p(x, y)$. Therefore, there exists $d<1$ such that $d^{2}=x^{2} / a^{2}+y^{2} / b^{2}$. Inside the adhesion surface, from (1) and (2),

$$
q_{\text {stick }}=\mu_{s} \frac{2 N}{\pi a b}\left(1-d^{2}\right) .
$$

The total tangential force component, $T$, is the sum of the integrals of $q(x, y)$ over the stuck and slip regions,

$$
T=\pi a b d^{2} q_{\text {stick }}+\mu_{k} N\left(1-d^{2}\right)^{2} .
$$

Equations (3) and (4) give a polynomial in $d^{2}$,

$$
\left(2 \mu_{s}-\mu_{k}\right) d^{4}-2\left(\mu_{s}-\mu_{k}\right) d^{2}=\mu_{k}-\frac{T}{N},
$$

with coefficients involving $\mu_{s}$ and $\mu_{k}$. This expression describes the dependency of the ratio, $d^{2}$, of the stuck region area to the total contact area on the ratio, $T / N$, of the tangential load component to the normal load component.

Only positive solutions not exceeding one have a physical meaning. When $\mu_{k}<\mu_{s}$, (5) has two solutions for the same $T / N$. Only the largest is realized since when $T / N$ is zero, $d=1$, that is, the stuck region coincides with the contact region. When $T / N$ rises from zero, $d$ decreases from one and thus must correspond to the greatest of the two solutions. The evolution proceeds until $T / N$ reaches a critical value, after which full slip begins, see Fig. 2 a. The critical size is $d_{\text {critic }}^{2}=\left(\mu_{s}-\mu_{k}\right) /\left(2 \mu_{s}-\mu_{k}\right)<1$. The ratio of the coefficients of friction can thus be estimated from $\mu_{k} / \mu_{s}=\left(1-d_{\text {critic }}^{2}\right) /\left(2-d_{\text {critic }}^{2}\right)$. If $\mu_{k}$ is assumed to be equal to $\mu_{s}$, (5) simplifies to $d^{2}=1-1 / \mu_{k} T / N$ which is similar to the previous model of Tada et al. (2006) with the difference that the right-hand-side is raised to the power $\frac{2}{3}$.

\subsection{Experiments}

We used the data of ten trials taken from the study of André et al. (2011) where subjects placed their index finger on a flat glass surface and applied a

\footnotetext{
${ }^{1}$ Movement before macroscopic sliding takes place (Johnson, 1955).
} 

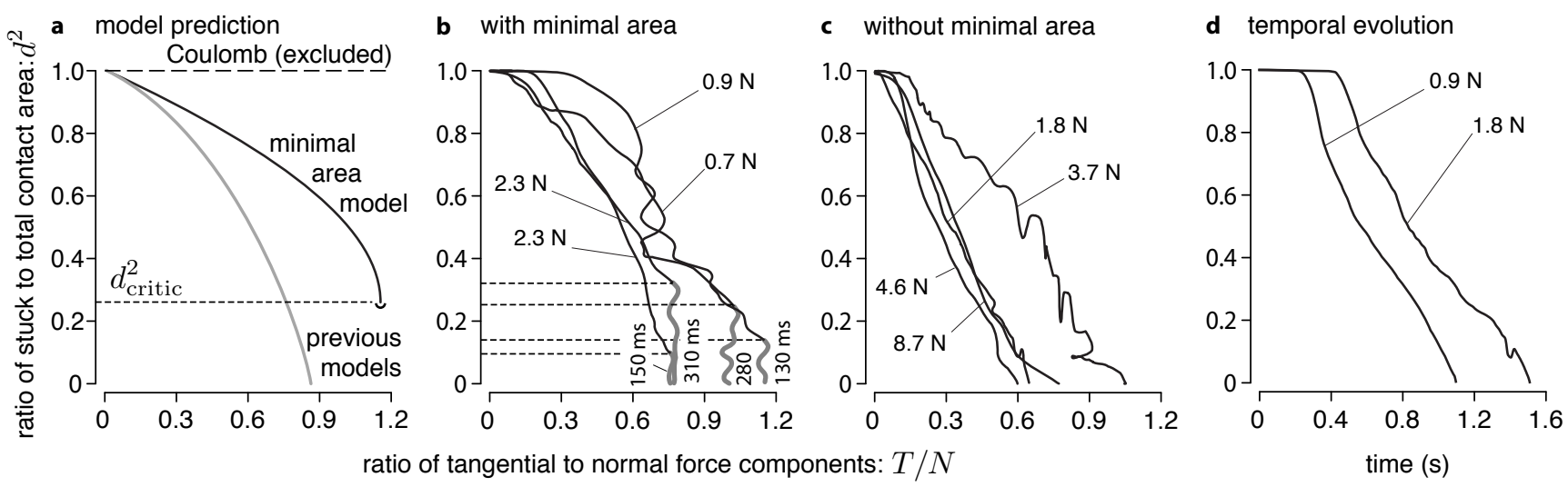

Figure 2: Dependency of the relative stuck area on the load. a) Standard model (gray line) and evolution model developed here (black line). The Coulomb model (dashed line) is excluded. b) Four experimental examples where the stuck area vanished based on the criterion that the ratio of the tangential to the normal force components stayed in a $\pm 5 \%$ corridor centered on its value before the complete loss of adhesion. The minimal adhesion surface area existed if the greatest value of $d^{2}$ inside the corridor was above 0.05 . The 130, 150, 280 and $310 \mathrm{~ms}$ temporal evolutions (thicker gray lines) cannot be predicted by a quasi-static model. The relative size of the critical area varied from 0.10 to 0.34 . c) Four other experimental trials where the stuck area diminished without meeting a critical size. d) Two examples of the temporal evolution of the stuck area, one from each category.

slowly ramping tangential load, maintaining the normal force to a constant value. The contact surface was imaged at $60 \mathrm{~Hz}$ as in (Levesque and Hayward, 2003 ) and the components of the interaction force recorded at $1.0 \mathrm{kHz}$. The area of the stuck region was estimated from optic flow processing applied between the first and the successive frames, using the algorithm described in (Bruhn et al., 2005), ${ }^{2}$ see Fig. 1, and caption.

\section{Results and Discussion}

Out of the ten trials inspected, four exhibited the existence of a minimal area and four did not. In the remaining two, the tangential component of the force of interaction was too unsteady for analysis. The two types of dependencies predicted by model (5) are illustrated in Fig. 2b and Fig. 2c. The oscillations seen in the curves were due to fluctuations of the force of

\footnotetext{
${ }^{2}$ Available at http://people.csail.mit.edu/celiu/OpticalFlow/
} 
interaction. These oscillations are not visible when the stuck surface area is plotted against time, as shown in Fig 2d.

The fact that a parabolic decay was not observed indicates that some of the assumptions made in Section 2.1 - chiefly among them are the constancy of the kinetic coefficient of friction on glass surfaces and the uniformity of the skin elasticity - may not hold. Nevertheless our model predicts the existence of two classes of solutions that are seen in the data.

If in some trials the stuck region vanished abruptly when measured against the ratio $T / N$ does not imply that it vanished instantaneously with respect to time. The time evolution lasted from $130 \mathrm{~ms}$ to $310 \mathrm{~ms}$. At the onset of this phase, the minimal adhesion surface area occupied from $10 \%$ to $34 \%$ of the total contact surface area (31\% to $58 \%$ in linear extent) and the tangential load component remained nearly constant thereafter.

Our experimental results show that partial slips with vanishing adhesion surfaces occur frequently. A gradual decrease of the adhesion surface area is nothing but a limiting case of the more general class of stick-to-slip evolutions with vanishing adhesion surfaces when the kinetic and static coefficient of friction are the same. Interestingly, the later case corresponds to very dry skin (Adams et al., 2007). Although skin hydratation data is available in our data, the sample set used is this communication is too small to establish reliable correlations between these observations, and so this question is left to future studies due to the complexities of skin tribology.

As far as we know, the notion of the existence of a minimal adhesion surface area has never been reported before. Classic works in the contact mechanics, such as (Johnson, 1955), do not consider the case of different kinetic and static friction coefficients, which is crucial for its existence, in a mathematical sense. Experimental studies, on the other hand, typically analyze the stuck region evolution in the temporal domain (Levesque and Hayward, 2003; Tada and Kanade, 2004; André et al., 2011), which prevents its observation since the temporal evolution has continuous time derivatives, as exemplified by Fig. 2d.

Finally, we would like to suggest that the existence of the minimal adhesion surface area in partial slips may play an important role during prehensile motor behavior. It is also reasonable to think that it may play a perceptual role, particularly in material identification and in the detection of small-scale surface irregularities. 


\section{Conflict of interest statement}

We wish to confirm that there are no known conflicts of interest associated with this publication and there was no significant financial support for this work that could have influenced its outcome.

\section{Acknowledgment}

The authors thank Vincent Levesque and Thibaut André for sharing the experimental data. This work was supported by an Advanced Grant of the European Research Council, agreement No. 247300.

\section{References}

Adams, M., Briscoe, B., Johnson, S., 2007. Friction and lubrication of human skin. Tribology Letters 26, 239-253.

André, T., Lévesque, V., Hayward, V., Lefèvre, P., Tonnard, J.L., 2011. Effect of skin hydration on the dynamics of fingertip gripping contact. Journal of the Royal Society Interface, on-line, doi: 10.1098/rsif.2011.0086.

Aoki, T., Niu, X., Latash, M.L., Zatsiorsky, V.M., 2006. Effects of friction at the digit-object interface on the digit forces in multi-finger prehension. Experimental Brain Research 172, 425-438.

Bruhn, A., Weickert, J., Schnörr, C., 2005. Lucas/Kanade meets Horn/Schunck: Combining local and global optic flow methods. International Journal of Computer Vision 61, 211-231.

Cole, K.J., Johansson, R.S., 1993. Friction at the digit-object interface scales the sensorimotor transformation for grip responses to pulling loads. Experimental Brain Research 95, 523-532.

Hayward, V., 2008. Haptic shape cues, invariants, priors and interface design. In Grunwald, M. (Ed.), Human Haptic Perception: Basics and Applications. Birkhäuser, Basel, pp. 381-392.

Johnson, K.L., 1955. Surface interaction between elastically loaded bodies under tangential forces. Proceedings of the Royal Society of London. Series A, Mathematical and Physical Sciences 230, 531-548. 
Levesque, V., Hayward, V., 2003. Experimental evidence of lateral skin strain during tactile exploration. In Proceedings of Eurohaptics, pp. 261-275.

Pawluk, D.T., Howe, R.D., 1999. Dynamic contact of the human fingerpad against a flat surface. Journal of Biomechanical Engineering 121, 605-611.

Smith, A.M., Gosselin, G., Houde, B., 2002. Deployment of fingertip forces in tactile exploration. Experimental Brain Research 147, 209-218.

Tada, M., Kanade, T., 2004. An imaging system of incipient slip for modelling how human perceives slip of a fingertip. In Proceedings of the 26th Annual Int. Conf. of the IEEE Engineering in Medicine and Biology Society, pp. 2045-2048.

Tada, M., Mochimaru, M., Kanade, T., 2006. How does a fingertip slip? visualizing partial slippage for modeling of contact mechanics. In Proceedings of Eurohaptics, pp. 415-420.

Witney, A.G., Wing, A., Thonnard, J.L., Smith, A.M., 2004. The cutaneous contribution to adaptive precision grip. Trends in Neurosciences 27, 637643.

Zatsiorsky, V.M., Latash, M.L., 2008. Multifinger prehension: an overview. Journal of Motor Behavior 40, 446-476. 